TITLE:

\title{
Sensitivity Analysis for L-Band Polarimetric Descriptors and Fusion for Urban Land Cover Change Detection
}

\author{
AUTHOR(S):
}

Mishra, Bhogendra; Susaki, Junichi

\section{CITATION:}

Mishra, Bhogendra ...[et al]. Sensitivity Analysis for L-Band Polarimetric Descriptors and Fusion for Urban Land Cover Change Detection. IEEE Journal of Selected Topics in Applied Earth Observations and Remote Sensing 2014, 7(10): 4231-4242

\section{ISSUE DATE:}

2014-10

URL:

http://hdl.handle.net/2433/193284

\section{RIGHT:}

(C) 2014 IEEE. Personal use of this material is permitted. Permission from IEEE must be obtained for all other uses, in any current or future media, including reprinting/republishing this material for advertising or promotional purposes, creating new collective works, for resale or redistribution to servers or lists, or reuse of any copyrighted component of this work in other works.; この論文は出版社版でありません。引用の際には出版社版をご確認ご利用ください。;This is not the published version. Please cite only the published version. 


\title{
Sensitivity Analysis for L-band Polarimetric Descriptors and Fusion for Urban Land Cover Change Detection
}

\author{
Bhogendra Mishra, Student Member, IEEE, and Junichi Susaki, Member, IEEE
}

\begin{abstract}
A fully Polarimetric Synthetic Aperture Radar (PolSAR) image allows the generation of a number of polarimetric descriptors. These descriptors are sensitive to changes in land use and cover. Thus, the objective of this study is twofold: first, to identify the most effective descriptors for each change type and ascertain the best complementary pairs from the selected polarimetric descriptors; and second, to develop an information fusion approach to use the unique features found in each polarimetric descriptor to obtain a better change map for urban and suburban environments. The effectiveness of each descriptor was assessed through statistical analysis of the sensitivity index in selected areas and through change detection results obtained by using the supervised thresholding method. A good agreement was found between the statistical analysis and the performance of each descriptor. Finally, a polarimetric information fusion method based on the coupling of modified thresholding with a region-growing algorithm was implemented for the identified complementary descriptor pairs. The mapping accuracy, as measured by the Kappa coefficient, was improved by 0.09 (from 0.76 to 0.85 ) with a significant reduction of false and missing alarm rates compared to using single polarimetric SAR images.
\end{abstract}

Index Terms - Synthetic aperture radar, Urban changes, Change detection algorithms, Polarimetric descriptors.

\section{INTRODUCTION}

I nformation on land use and cover and the changing patterns of these data is always a hot topic because of its importance in several applications, including land policy development, site selection, and demographic and environmental issues at the national, regional, and global scale. Collecting the change information by ground-based survey is more accurate than any other method, but this is impractical to do regularly and at short intervals in a rapidly growing urban area. Thus, remote sensing is the best available technique to monitor these changes. The utility of synthetic aperture radar (SAR) images in change detection has already been proven for urban change detection and disaster monitoring [1]-[4]. However, the full potential of Polarimetric SAR (PolSAR) images still needs to be explored.

The use of radar intensity information has been suggested for change detection rather than correlation coefficients and phase differences between co-polarized channels [5]. Several studies have been published based on radar intensities [1]-[6], but most of these studies were based on single polarimetric images rather than fully polarimetric images [1], [4], [6]. A fully polarimetric image allows the development

The authors are with Department of Civil and Earth Resources Engineering, Geoinformatics lab, Kyoto University, Japan (corresponding author e-mail: mishra.bhogendra.46c@st.kyoto-u.ac.jp). of several descriptors by different image processing algorithms. The representation and fundamental methods are based on incoherent analysis, which works with an ensemble average of several pixels to give second order statics of polarimetric information [7]. This allows the generation of several very useful pieces of information and descriptors [8], [9]. These descriptors could supplement the results derived from single polarization images by adding several unique features, reflecting the complex nature of man-made structures, that could be sensitive to different types of changes in urban environments.

The availability of several processing techniques and the possibility of generating several descriptors from fully PolSAR images have created a good opportunity to conduct a sensitivity study to find the most effective descriptors for detecting several types of change. In this study, we also identify the best pairs of complementary components of these polarimetric descriptors. Furthermore, we develop an information fusion approach to combine information from the unique features found in each polarimetric descriptor in order to generate a better change map for urban and suburban environments.

The data used and study area are described in Section II. Section III contains an explanation of the methodology followed in this study. In the first part, we discuss the processing technique used to generate the polarimetric descriptors, and in the second part, we discuss the proposed polarimetric fusion technique. The results are presented and discussed in Section IV. The discussion of the results includes the sensitivity analysis and results obtained from the fusion of selected complementary pairs of polarimetric descriptors. Finally, our conclusions are given in Section V.

\section{II.DATA USED AND STUDY AREA}

Ho Chi Min City, one of the fastest growing Asian cities, was selected for the study. Its location is shown in Fig. 1. To detect changed areas, two fully polarimetric images acquired by the Advanced Land Observing Satellite (ALOS) Phased Array type L-band Synthetic Aperture Radar (PALSAR) in April 2007 and April 2011 were used. To reduce the effects of phenological changes in vegetation and water content on the land surface, the images were acquired at nearly the same time of year. Additionally, both years selected had a normal precipitation pattern. Thus, all the changes detected in multi-temporal images are assumed to be related to human activity. To confirm all possible types of change, a section of approximately $18 \mathrm{~km} \times 45 \mathrm{~km}$ pixels that includes a core urban area and a rapidly expanding suburban area, was selected. To assess the accuracy of our approach, Advanced Visible and Near Infrared Radiometer type-2 (AVNIR-2) images 


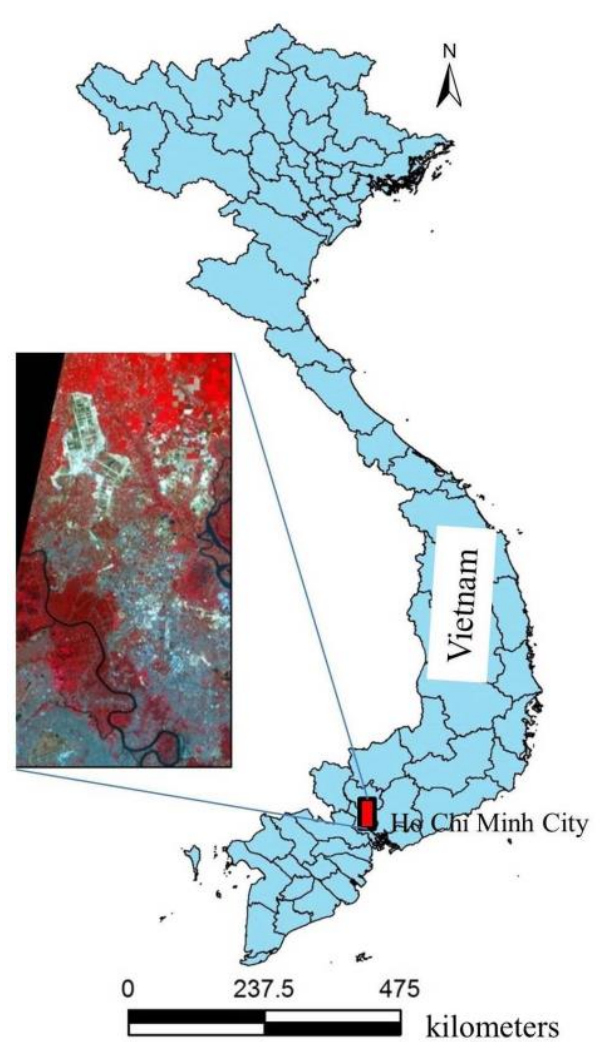

Fig. 1. Study area.

acquired on nearly the same dates as the PALSAR images were used. In addition, high-resolution QuickBird images from Google Earth were used as a supplementary source to develop a reference change map.

\section{Methodology}

The process flow diagram of the sensitivity analysis and the fusion of polarimetric descriptors in multi-temporal PolSAR images is presented in Fig. 2. Details of the methodology are given in the following sub-sections.

\section{A. Generation of Polarimetric Descriptors and Preprocessing}

Several polarimetric decomposition methodologies are available to extract the physical scattering mechanism. In this study, we use descriptors obtained from different stages of the polarimetric processing: four polarimetric components $(\mathrm{HH}, \mathrm{HV}, \mathrm{VH}$, and $\mathrm{VV})$, the diagonal elements of the coherency matrix $\left(\mathrm{T}_{11}, \mathrm{~T}_{22}\right.$, and $\left.\mathrm{T}_{33}\right)$, the eigenvector-based descriptors alpha $(\alpha)$, and Entropy $(H)[8]$, and three physical scattering components, surface scattering $\left(\mathrm{P}_{\mathrm{S}}\right)$, double bounce $\left(\mathrm{P}_{\mathrm{D}}\right)$, and volume scattering $\left(\mathrm{P}_{\mathrm{V}}\right)$ [9].

The PolSAR data consist of complex scattering values, which can be represented by the $2 \times 2$ scattering matrix shown in Eq. (1).

$$
S=\left(\begin{array}{ll}
S_{H H} & S_{H V} \\
S_{V H} & S_{V V}
\end{array}\right)=\left(\begin{array}{ll}
a & b \\
b & c
\end{array}\right) .
$$

For mono-static radar imaging of a reciprocal medium, we have $\mathrm{S}_{\mathrm{HV}}=\mathrm{S}_{\mathrm{VH}}[7]$.

In a multilook PolSAR image, each pixel is represented by a $3 \times 3$ coherency matrix

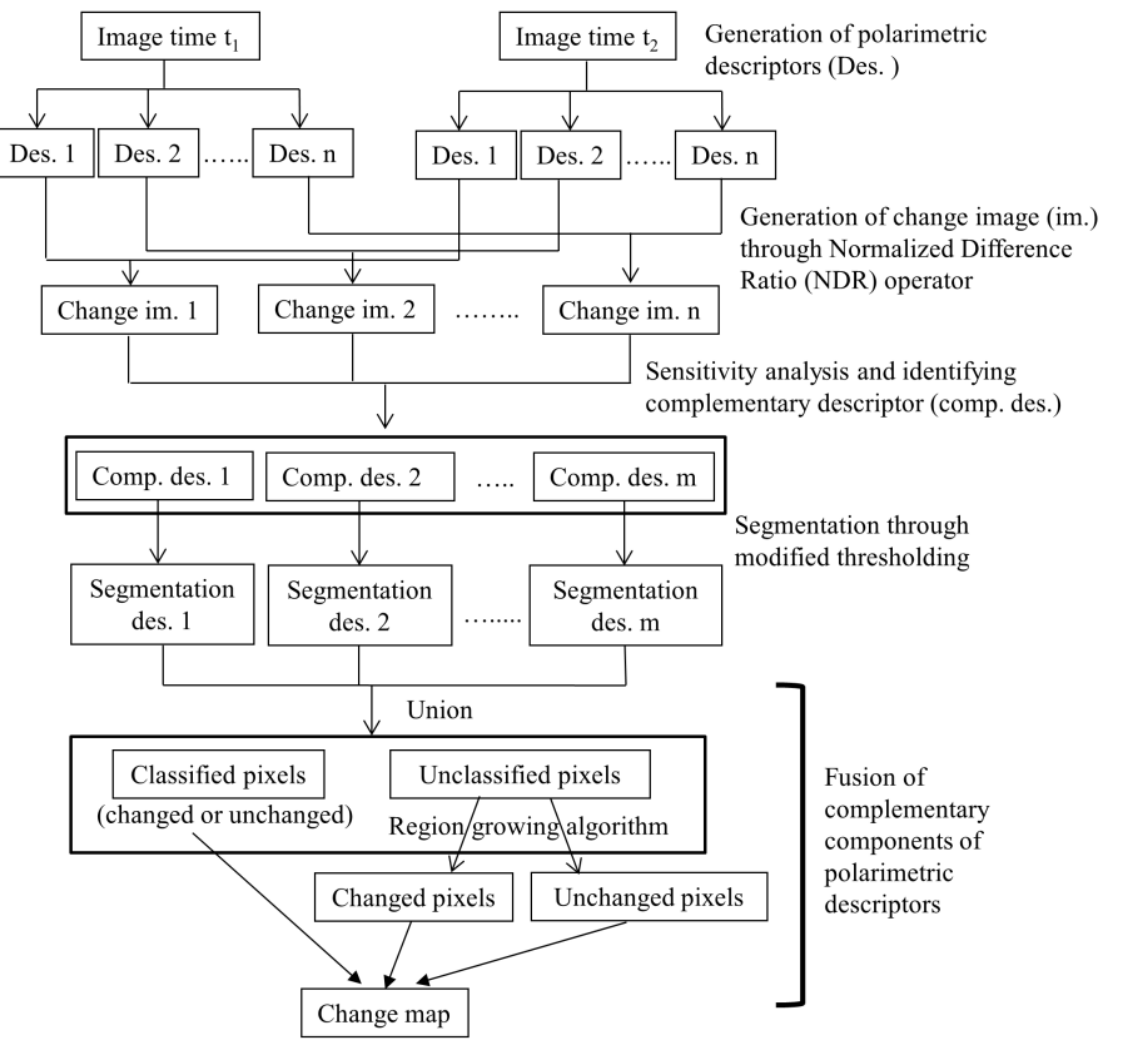

Fig. 2. Process flow diagram.

$$
\begin{aligned}
T & =\left(\begin{array}{lll}
T_{11} & T_{12} & T_{13} \\
T_{21} & T_{22} & T_{23} \\
T_{31} & T_{32} & T_{33}
\end{array}\right) \\
= & \frac{1}{2}\left(\begin{array}{ccc}
|a+b|^{2} & (a+b)(a-b)^{*} & 2(a+b) c^{*} \\
(a-b)(a+b)^{*} & |a-b|^{2} & 2(a+b) c^{*} \\
2 c(a+b)^{*} & 2 c(a-b)^{*} & 4|c|^{2}
\end{array}\right)
\end{aligned}
$$

where * represents the complex conjugate operation.

The value of $H$ obtained from a Cloud and Pottier alpha-entropy $(\alpha-H)$ decomposition of the coherency matrix [8] defines the roughness of the scattering: $H=0$ indicates a single scattering mechanism, while $H=1$ indicates a random mixture of scattering mechanisms. The scattering angle $(\alpha)$, representing the mean scattering mechanism, is a continuous angle ranging from $0^{\circ}$ to $90^{\circ}$. In a model-based approach, the scattering matrix is decomposed into the physical scattering components $\mathrm{P}_{\mathrm{S}}, \mathrm{P}_{\mathrm{D}}$, and $\mathrm{P}_{\mathrm{V}}$ [9].

All generated descriptors were geocoded and co-registered to the Universal Transverse Mercator (UTM) system using the Global Digital Elevation Model (GDEM) with $30 \mathrm{~m}$ pixel spacing using ASF MapReady 3.2 [10]. In order to reduce the speckle noise present in the SAR image, Enhanced Lee filter [11] of window size $5 \times 5$ was implemented. The window size was selected with caution, if the size is bigger the spatial resolution will lose and if the window size is smaller, the filter will not be effective [1], [12].

\section{B. Change Image Development}

The Normalized Difference Ratio (NDR) operator is used to generate the change image. The NDR operator is defined as: 


$$
\text { change }=\frac{x_{2}-x_{1}}{x_{2}+x_{1}},
$$

where, $x_{1}$ and $x_{2}$ are the SAR backscattering magnitude at two dates, $t_{1}$ and $t_{2}$, obtained from co-registered images. This operator was introduced by Coppin and Bauer in [13] and later used on optical images in [14] and on SAR images in [12]. The NDR operator is better for change detection than other traditional operators [12], [14] because the NDR image has less errors than an image produced by difference or ratio operator. We now compare the errors generated from NDR and ratio operator. For the easeness the Eq. (3) can be rewritten as follows:

$y=\frac{x_{2}-x_{1}}{x_{2}+x_{1}}$,

where, $y$ is the change image generated from NDR operation. According to the law of error propagation, the variance of NDR operator, $\sigma_{y}^{2}$, can be derived as follows:

$\sigma_{y}{ }^{2}=\left\{\frac{d}{d x_{1}}\left(\frac{x_{2}-x_{1}}{x_{2}+x_{1}}\right)\right\}^{2}{\sigma_{x_{1}}}^{2}+\left\{\frac{d}{d x_{2}}\left(\frac{x_{2}-x_{1}}{x_{2}+x_{1}}\right)\right\}^{2} \sigma_{x_{2}}{ }^{2}$

$\sigma_{y}^{2}=\frac{4 x_{2}^{2}}{\left(x_{2}+x_{1}\right)^{4}} \sigma_{x_{1}}{ }^{2}+\frac{4 x_{1}^{2}}{\left(x_{2}+x_{1}\right)^{4}} \sigma_{x_{2}}{ }^{2}$.

Assume that the variance of $x_{1},\left(\sigma_{x_{1}}^{2}\right)$, and $x_{2},\left(\sigma_{x_{2}}^{2}\right)$ are equal and substitute them by $\sigma_{x}^{2}$.

$\frac{\sigma_{y}^{2}}{\sigma_{x}^{2}}=\frac{4\left(x_{2}^{2}+x_{1}^{2}\right)}{\left(x_{2}+x_{1}\right)^{4}}$.

Similarly, the ratio operation is defined as:

$z=\frac{x_{2}}{x_{1}}$,

where, $z$ is the image generated from ratio operation, $x_{1}$ and $x_{2}$ are the backscattering magnitude at two dates According to the law of error propagation, the variance of ratio operator can be calculated as follows:

$\sigma_{z}^{2}=\left\{\frac{d}{d x_{1}}\left(\frac{x_{2}}{x_{1}}\right)\right\}^{2} \sigma_{x_{1}}^{2}+\left\{\frac{d}{d x_{2}}\left(\frac{x_{2}}{x_{1}}\right)\right\}^{2} \sigma_{x_{2}}{ }^{2}$

$\sigma_{z}^{2}=\frac{x_{2}^{2}}{x_{1}{ }^{4}} \sigma_{x_{1}}{ }^{2}+\frac{1}{x_{1}{ }^{2}} \sigma_{x_{2}}^{2}$

$\sigma_{z}^{2}=\frac{x_{2}^{2} \sigma_{x_{1}}^{2}+x_{1}^{2} \sigma_{x_{2}}^{2}}{x_{1}{ }^{2}}$.

Similar to the NDR operation, $\sigma_{x_{1}}^{2}$ and $\sigma_{x_{2}}^{2}$ are assumed to be equal and substituting them by $\sigma_{x}^{2}$ and gives:

$\frac{\sigma_{Z}^{2}}{\sigma_{x}^{2}}=\frac{\left(x_{2}^{2}+x_{1}^{2}\right)}{x_{1}^{4}}$.

Dividing Eq (5) by Eq (7) gives:

$\frac{\sigma_{y}{ }^{2}}{\sigma_{x}{ }^{2}} \times \frac{\sigma_{x}{ }^{2}}{\sigma_{z}{ }^{2}}=\frac{4\left(x_{2}{ }^{2}+x_{1}^{2}\right)}{\left(x_{2}+x_{1}\right)^{4}} \times \frac{x_{1}{ }^{2}}{\left(x_{2}{ }^{2}+x_{1}{ }^{2}\right)}$, $\frac{\sigma_{y}^{2}}{\sigma_{z}^{2}}=\frac{4 x_{1}^{4}}{\left(x_{2}+x_{1}\right)^{4}}$

The right hand side of Eq. (8) is less than 1 for all value of $x_{2}$ $>0.414 x_{1}$. This suggests that the variance of ratio operator is smaller than the variance of NDR operator only if the backscattering intensity is decreased heavily i.e $x_{2}<0.414 x_{1}$. This fact is presented in the Fig. 3 generated by plotting the contour line using Eq. (8). It is shown that a very small area has the ratio higher than 1 , that means ratio operator is better than NDR in very limited combination of $x_{1}$ and $x_{2}$. As the probability of error in detecting changes increases with the variance [15], the NDR method produces less error than the ratio method. Therefore, NDR operator has been adopted in this study.

The input SAR images have a Gamma distribution and it approximates to the Gaussian distribution while increasing the number of look. Given a change area in an input images, the image obtained by the ratio and NDR operator is a multi-mode image. Thus, a joint distribution is necessary. Regarding the probability density function (PDF) of such multi-model images, the authors in [2] performed a comparative study of Nakagami, Lognormal and Weibull distribution for ratio images, and concluded that all have comparable results. Similarly, the no-change area is better modeled with Gaussian distribution than the Logistic and Student's $t$ distribution with the image generated by the NDR operator [12].

\section{Sensitivity Analysis}

Two approaches were considered for sensitivity analysis: the first is the analysis of separability index for several selected changed areas for each polarimetric descriptor; the second is change mapping using a single polarimetric descriptor.

1) Separability Index

The separability index of a descriptor indicates the degree of separability of that descriptor. It is computed from Eq. (9) [16].

$$
\text { Separability_index }=\frac{\left|\mu_{c}-\mu_{u c}\right|}{\sigma_{c}+\sigma_{u c}} \text {. }
$$

Here, $\mu_{\mathrm{c}}$ and $\sigma_{\mathrm{c}}$ are the mean and standard deviation of the change class, and $\mu_{\mathrm{uc}}$ and $\sigma_{\mathrm{uc}}$ are the mean and standard deviation of the no-change class. The descriptor with the

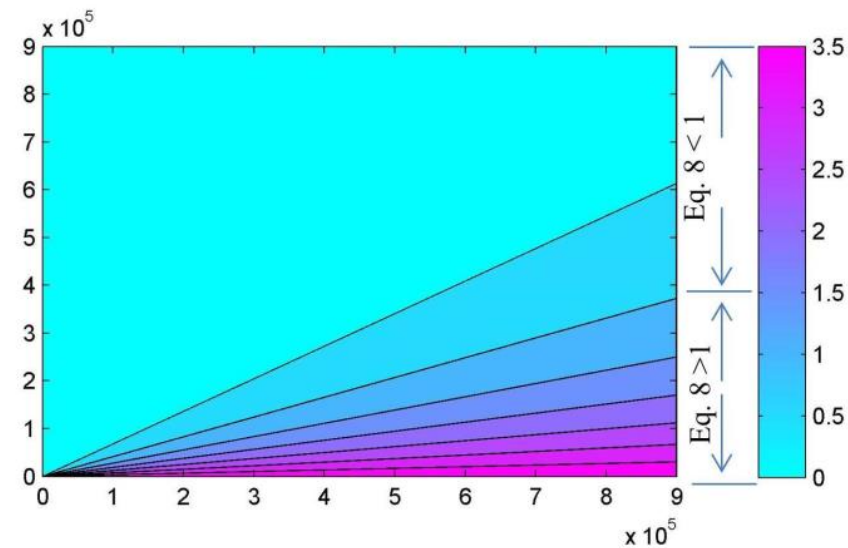

Fig. 3. Contour map of ratio of error propagation in NDR and ratio image. 
highest separability index is the best to use for change detection. In order to compute the separability index, a prior knowledge of the change area is required. Hence, four major change classes - bare land to built-up, agriculture to built-up (under construction), deforestation, and agricultural to bare land (smoothing of agricultural land) - were identified with reference to the high-resolution AVNIR-2 optical images and analyzed.

\section{Change Mapping Through Supervised Thresholding} Using a Single Polarimetric Descriptor

By assuming a Gaussian distribution of no-change areas in the change image generated by the NDR operator, a threshold value was identified in a supervised manner. The NDR image allows a clear preliminary assessment of changes by simple visual inspection [14]. Fig. 4 shows the NDR image for the $\mathrm{HH}$ polarimetric component. Bright areas indicate increased backscattering intensity, whereas dark areas indicate decreased backscattering intensity. The majority of the area is moderately bright or dark and is composed of those pixels having values around 0 . This area is characterized as the no-change (smooth) area. Thus, it is easy to recognize clusters of no-change area by visual inspection (Fig. 4).

The basic premise in using remote sensing data for change detection is that changes in land cover must result in changes in reflectance values and changes in reflectance due to land cover change must be large with respect to reflectance changes caused by other factors [17]. However, in the case of SAR images, it is the result of changes in backscatter and changes in the backscatter value due to the land cover change must be larger than the changes in backscatter caused by other factors.

As the no-change area has a Gaussian distribution, the range $\mu \pm 3 \sigma$ covers almost all pixels $(99.7 \%$ of the total sample), and the rest is assumed to be noise. Therefore, it is clear that expanding the threshold value (e.g. $\mu \pm 4 \sigma$ ) will not increase inclusion of the no-change pixel considerably. With reference to the basic premise, none of the change pixels fall in the range of no-change area i.e. $\mu \pm 3 \sigma$. If we narrow down the threshold value, change pixels do not fall in that range, eventually increase the missing alarm of no-change area and false alarm of change area. For example, if narrow down the threshold value to $\mu \pm 2 \sigma, 4 \%$ of the no-change pixels classify as the change area. Therefore, $\mu \pm 3 \sigma$ would be the ideal threshold value to segment the no-change area from change and adopt in this study.

Several sample no-change clusters in the change image are needed to select manually. If the sample no-change area has mean $(\mu)$ and standard deviation $(\sigma)$, the threshold values can be computed as:

$$
\text { Threshold }=\left\{\begin{array}{c}
\text { Left Threshold }\left(t_{1}\right)=\mu-3 \sigma \\
\text { Right Threshold }\left(t_{2}\right)=\mu+3 \sigma
\end{array} .\right.
$$

The advantage of this method is that no assumption is required about change areas, which is unpredictable. This method appears to give nearly the same results as the manual trial-and-error procedure, but it depends on the selected sample [12].

With the inherent problem of the supervised methodology, the accuracy of this method depends on the selected sample. However, due to the implementational advantage of the image generated by NDR operator, that allows a clear

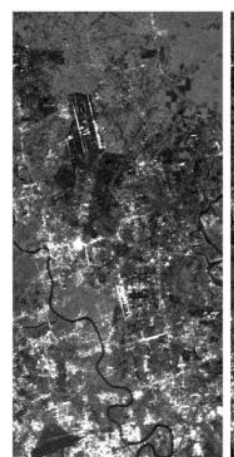

(a)

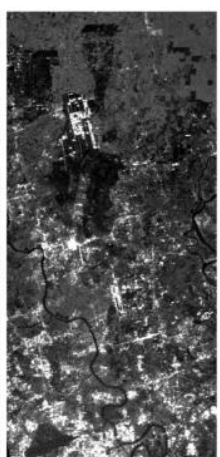

(b)

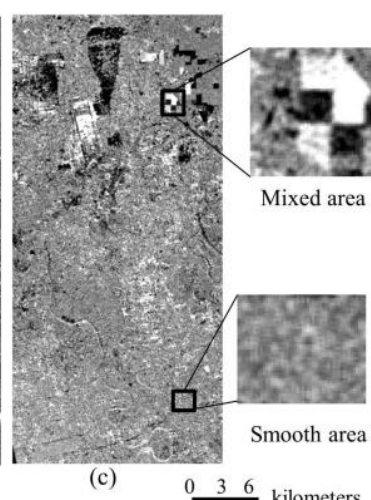

(c)
Fig. 4. Change image generation using the NDR operator. HH component of PolSAR images (a) taken in 2007, (b) taken in 2011 and (c) the change image.

preliminary assessment of the change area by simple visual inspection [14], a sufficiently large number of sample no-change pixels are possible to select. Thus, the threshold range is unlikely to shift significantly.

A change in the land use or cover pattern alters the backscattering mechanism, and thus changes the backscattering intensity. For example, changing from bare land to a built-up area changes the backscattering mechanism from surface to double bounced reflection, which causes an increase in backscattering intensity. Similarly, deforestation changes the backscattering mechanism from volume to surface reflection, thus decreasing the backscattering intensity. Because such backscattering mechanisms are known for each feature, we assume the terms "increase in backscattering intensity" and "decrease in backscattering intensity" always have the same meaning for different features. However, this assumption may not be valid for some descriptors such as $\mathrm{P}_{\mathrm{s}}, \alpha$ and $\mathrm{H}$. Changes in pixels located beyond the left threshold obtained from Eq. (10) are attributed to a decrease in backscattering intensity and those located beyond the right threshold are attributed to an increase in backscattering intensity.

\section{E. Polarimetric Fusion by Considering Spatial Information for Change Detection}

We propose the fusion of several descriptors, to be effected by coupling thresholding with a region-growing algorithm that considers spatial information.

If we have threshold values $t_{1}$ and $t_{2}\left(t_{1}<t_{2}\right)$ obtained from Eq. (10), we can compute the mean $(\mu)$ and standard deviation $(\sigma)$ of values falling within this threshold range. It is empirically found that a majority of false alarms come from $t_{1} \pm \sigma$ or $t_{2} \pm \sigma$; that is, from the overlap of the histograms of the change and no-change classes, as shown in Fig. 5. Thus, the threshold values were modified to mitigate this effect. The pixels in the range $t_{1}+\sigma$ to $t_{2}-\sigma$ are classified as belonging to the no-change group. Similarly, pixels with value less than $t_{1}-\sigma$ are classified as having a decrease in intensity and those with value greater than $t_{2}+\sigma$ are classified as having an increase in intensity. The remaining pixels, from $t_{1}-\sigma$ to $t_{1}+\sigma$ and from $t_{2}-\sigma$ to $t_{2}+\sigma$ remained unclassified [12].

The fusion process is explained here for the two descriptors case. This operation was carried out in two steps. First, the union operation was performed for all pixels classified by both descriptors; this operation is illustrated in 
Fig. 6. At this stage, we have two types of pixels: classified

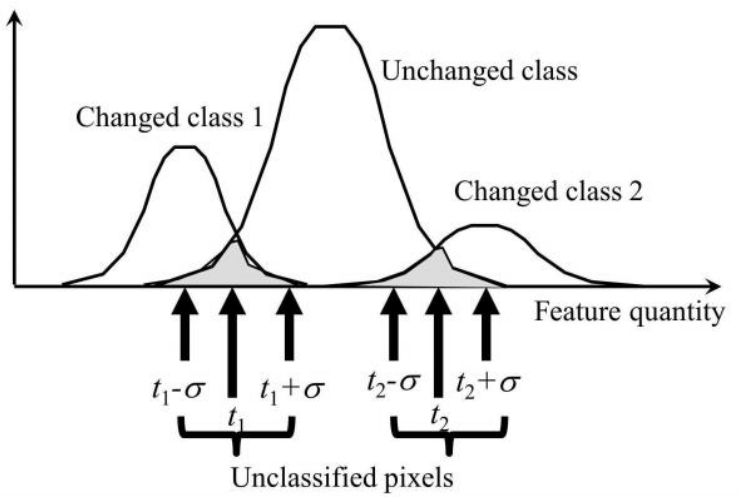

Fig. 5. Modified thresholding. The two threshold values are $t_{1}$ and $t_{2}$, and $\sigma$ is the standard deviation in the unchanged class $\left(t_{1}\right.$ to $\left.t_{2}\right)$. The regions $t_{1} \pm \sigma$ and $t_{2} \pm \sigma$, in which two classes overlap (dark area), are left unclassified after modifying the threshold values.

and unclassified. The classified pixels are a member of one of three classes $C_{1}, C_{2}$, or $C_{3}$, which represent no change, increased intensity, and decreased intensity, respectively. The classified pixels are treated as seed pixels, after which unclassified pixels are classified by a region-growing approach that operates locally.

Formally, let $U$ represents the set of unclassified pixels. For all $x$ in $U$, compute the distance $\left(\Delta x_{i}\right)$ from each connected class as follows:

$$
\Delta x_{i}=\left|g(x)-g_{i}(c)\right|
$$

where, $i=1,2$, or $3, g(x)$ is the value of pixel $x$, and $g_{i}(c)$ is the average pixel value for each class. The connected class members are obtained from two time dilations of $x$.

We next obtain $\Delta x_{i}$ for each descriptor to be fused. For descriptor 1, decp1Inc, decp1Dec, and decp 1 Noc are the distances of the pixel $x(i, j)$ to the increased intensity class, the decreased intensity class and the no-change class, respectively. Similarly, for descriptor 2, decp2Inc, decp2Dec, decp2Noc, are the distances to the increased intensity class, the decreased intensity class and the no-change class, respectively. Now, the overall distances of all classes from $x(i, j)$, considering both descriptors, is computed as follows.

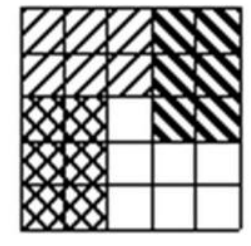

(a) Descriptor 1

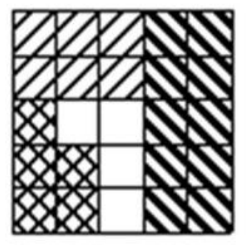

(b) Descriptor 2

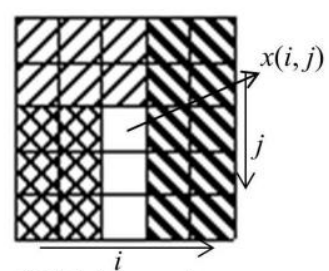

(c) Union results

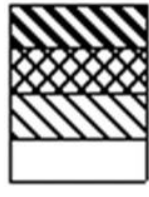

Increase intensity

Decrease intensity

No change

Unclassified

Fig. 6. Union operation of classified changed areas obtained from modified thresholding in a complementary pair of polarimetric descriptors. (a) Descriptor 1, (b) descriptor 2, and (c) results obtained by the union of areas classified in (a) and (b). Three pixels, $x(i, j), x(i, j+1)$ and $x(i, j+2)$, are unclassified by both descriptors.

$$
\left.\begin{array}{l}
\text { distInc }=\operatorname{dec} 1 \operatorname{Inc}+\operatorname{decp} 2 \operatorname{Inc} ; \\
\text { distDec }=\operatorname{dec} 1 \mathrm{Dec}+\operatorname{dec} 2 \mathrm{Dec} ; \\
\operatorname{distNoc}=\operatorname{dec} 1 \mathrm{Noc}+\operatorname{decp} 2 \mathrm{Noc}
\end{array}\right\} .
$$

Here, distInc is the overall distance of pixel $x(i, j)$ to the increased intensity class, distDec is the overall distance of $x(i$, $j$ ) from the decreased intensity class, and distNoc is the overall distance of $x(i, j)$ from the no-change class.

Each unclassified pixel is assigned to the class that has the minimum distance from the pixel. This classification is similar to the $k$-minimum distance classifier. The process will be repeated as long as there is an unclassified pixel with at least two neighboring classes. The remaining pixels will be classified as no-change. This method can be extended to more than two descriptors in an analogous manner.

\section{RESULTS AND DISCUSSION}

The assumption of the null hypothesis, Gaussian distribution in no-change area, was verified with normal probability curve. And the effectiveness of the supervised thresholding was verified by comparing the performance with a parametric approach (minimum error thresholding) [6] and a non-parametric approach (Otsu thresholding) [20].

Given that the study area has the positive (increased backscattering intensity) and negative changes (decreased backscattering intensity). The resulting image generated through NDR operator will have multi-model histogram; therefore, theoretically derived PDF will not be able to model such dataset. Many previous work with such kinds of datasets assumed a joint distribution and performed a statistical test for each area (changed and no-changed) in order to identify the approximate distribution. For example: in paper [1] a generalized Gaussian distribution was assumed and verified with the statistical test while implementing the logratio operator. The paper [2] concluded that three non-Gaussian distributions, Nakagami, Log-normal, and Weibull distribution for ratio image of the amplitude values of SAR images have similar performance. In another work [3] Gaussian distribution was assumed in both change and no-change area while using the difference operator and continuing without any test. Similarly, in [6] Nakagami and lognormal distributions are recommended in the image generated from modified ratio operator. Likewise, in order to test the null hypothesis that no-change areas are normally distributed, an NDR image generated from the $\mathrm{HH}$ component was subjected to analysis in this study.

The study area was divided into three subzones. The experiment was performed for four datasets, one for the whole study area and each of the three subzones. A normal probability curve fitting method was used to verify the Gaussian distribution in the sample no-change area selected from each zone. The results are presented in Figs. 7(a)-(d). Further, to show the range of $\mu \pm 3 \sigma$ within the no-change area with respect to all areas, a Gaussian PDF is also shown for each zone (Figs. 7(f)-(i)). By using the same sample no-change pixels, threshold values were obtained for each zone; the change results are shown in Figs. 7(j)-(m). The results in all zones are very consistent and reasonable. From this analysis, it is clear that the no-change area can be stably approximated with a Gaussian distribution. Thus, the null 

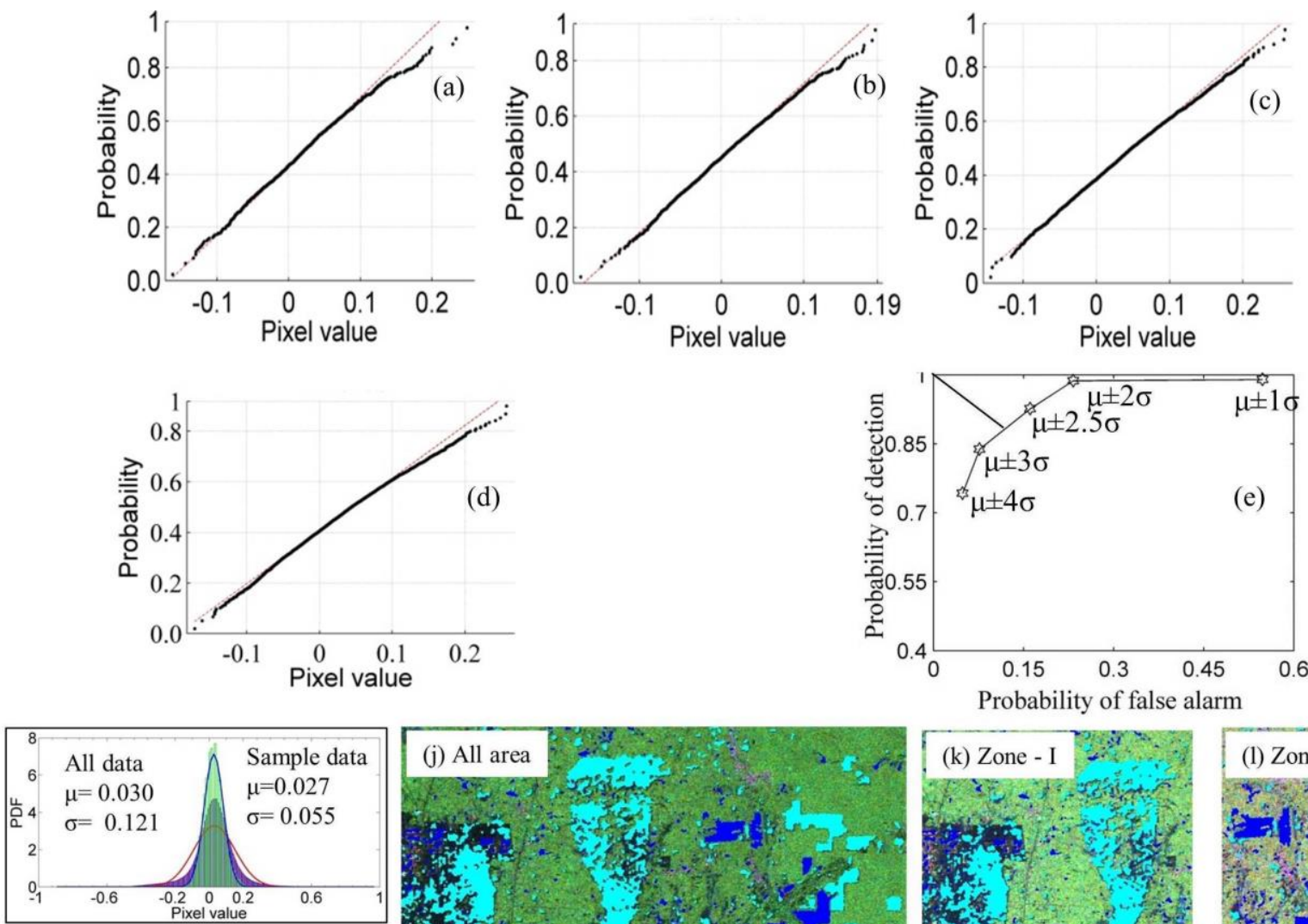

(f) All area

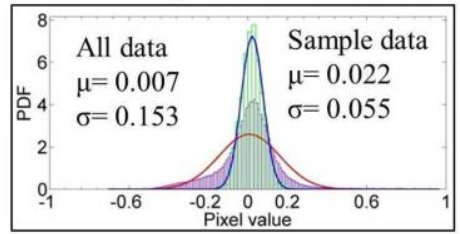

(g) Zone - I

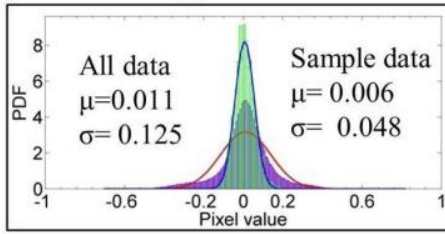

(h) Zone - II

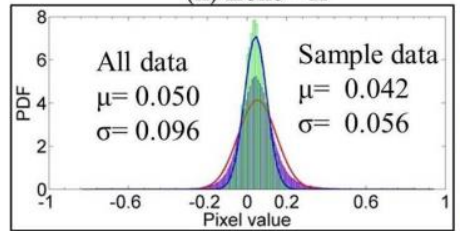

(i) Zone - III

-All data
- Gaussian for all data
— Sample no-change data
— Gaussian for sample no-change data

$$
\begin{array}{llll}
0 & 1 & 2 & 4 \\
\hline
\end{array}
$$
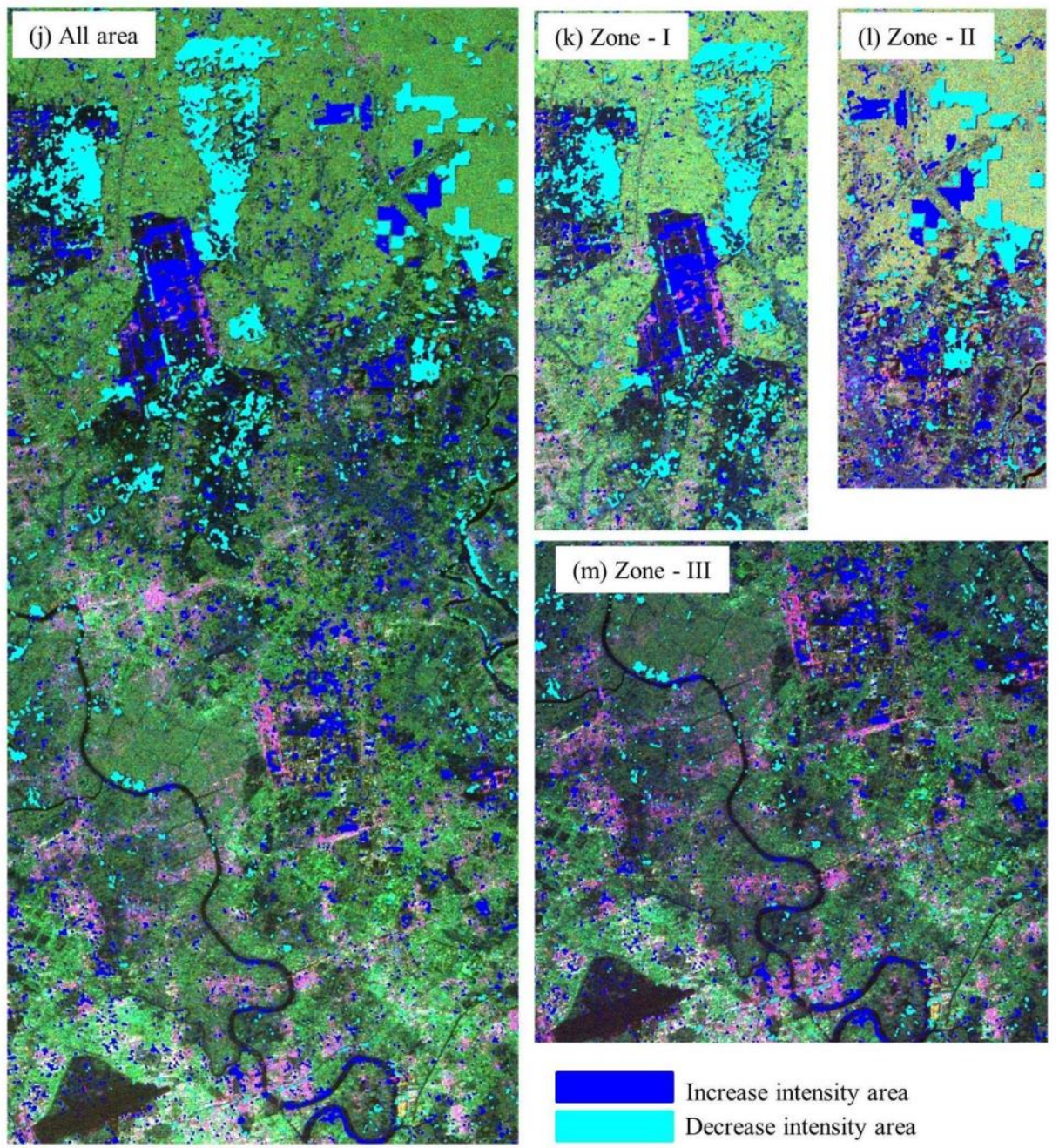

Fig. 7. Test results for Normal distribution. (a) - (d) Normal probability curve for sample no-change area in each selected zone, (e) ROC curve with various thresholding values in all area presented in (j), (f) - (i) Gaussian PDF fitting for sample no-change area and whole area in each zone and $(\mathbf{j})-(\mathrm{m})$ corresponding change results in each zone based on the threshold value obtained from the sample no-change pixels, overlaid with Pauli vector false color composite. 
hypothesis was not rejected for the $\mathrm{HH}$ component. We assume that other polarimetric descriptors also have the same statistical distribution. A detailed analysis of this approach is presented in [12].

In order to confirm the appropriate thresholding value, a receiver operating characteristic (ROC) curve was generated with five different thresholding values $(\mu \pm 4 \sigma, \mu \pm 3 \sigma, \mu \pm 2.5 \sigma$, $\mu \pm 2 \sigma$, and $\mu \pm 2.5 \sigma$ where $\mu$ is the mean and $\sigma$ is the standard deviation in the sample no-change area) and presented in Fig. 7. (e). The threshold value, which generates the closest point in the ROC curve from the upper left corner $(0,1)$ (having the highest probability of detection and the lowest probability of false alarm) is the best to segment the change area from no-change area [21]. From the visual analysis, it is inferred that, some value in between $\mu \pm 3 \sigma$ and $\mu \pm 2.5 \sigma$ could give the best results. The point is very close to the $\mu \pm 3 \sigma$ and that does not make a significant difference in the results with respect to the $\mu \pm 3 \sigma$ (Fig. 7(e)). Therefore, the assumption of $\mu \pm 3 \sigma$ as a threshold value (Eq. 10) to segment the no-change background from change area is valid.

Similarly, Fig. 8 (a) shows the change map obtained from the manual trial and error procedure (MTEP) thresholding, Fig. 8 (b) from supervised thresholding, Fig. 8 (c) from minimum error thresholding and Fig. 8 (d) from Otsu thresholding. The Kappa indices are 0.76, 0.74, 0.70 and 0.71 for MTEP, supervised method, minimum error algorithm and Otsu thresholding algorithm, respectively. The results obtained from minimum error thresholding has the lowest accuracy. The big missing alarm in both increased and decreased backscattering areas was identified, though the majority of error comes from decreasing backscattering area. While implementing the Otsu thresholding, it omits a large decrease backscattering area and gained a commission in an increased backscattering area. The major reasons behind the poor performance in minimum error are two folds: one is due to the inappropriate assumption of the same statistical distribution in both change and no-change classes, and the other is the uses of single thresholding values for detecting two types of changes (increased and decreased backscattering). The Otsu thresholding approach also suffers from the same problem. When converting data into a single-tail statistic, the nature of the curve will differ from both tails and a single threshold value cannot perfectly judge both types of change. That is, what is good for one type of change (increased or decreased backscattering) will not be good for the other. In this case, a higher missing alarm rate in detecting decreased backscattering areas and false alarm in

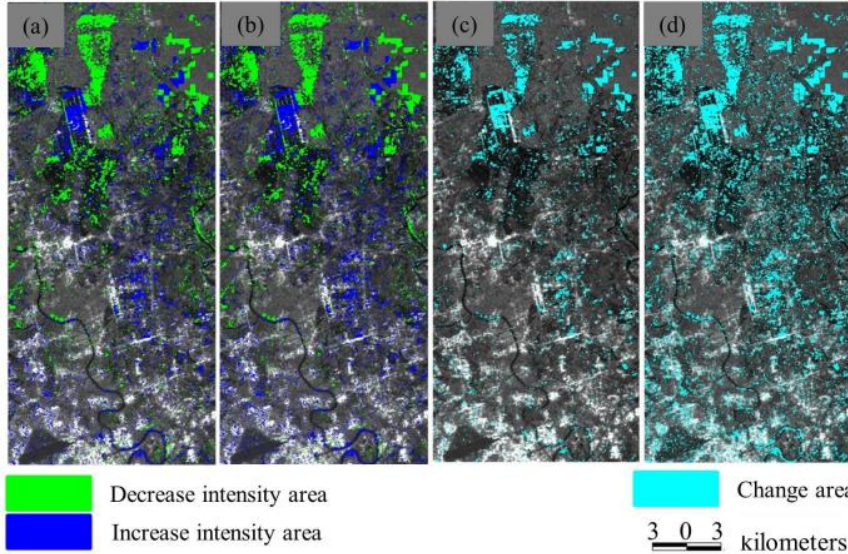

Fig. 8. Change mapping using several methods - (a) MTEP, (b) supervised, (c) minimum error thresholding and (d) Otsu thresholding.

the increased backscattering area were identified even though the thresholding values obtained from Otsu algorithm is reasonable. In order to solve such problems: one tail problem, and the same distribution for change and no-change areas, a supervised thresholding methodology appear to be effective among three approaches. Therefore, we adopt the supervised thresholding method for sensitivity analysis and as a pre-processing for polarimetric fusion process.

\section{A. Sensitivity Analysis}

\section{1) Separability Analysis}

A separability index was computed from Eq.(9) for each major change classes (agriculture to under construction area, bare land to built-up area, deforestation, and agriculture to bare land area) across all considered polarimetric descriptors. The NDR images of each descriptor obtained from multi-temporal PolSAR images were subjected to analysis. Fig. 9 shows the separability index for each descriptor in the selected change classes. Higher separability indexes indicate a better ability to detect changes.

In Fig. 9, it can be clearly seen that $\mathrm{P}_{\mathrm{V}}, \mathrm{HV}$, and $\mathrm{T}_{33}$ have very similar indexes and are the highest in three change classes: agriculture to bare land (surface smoothing), construction, and deforestation. However, they are not good indicators for the change from bare land to built-up area. Some other descriptors appear better for detecting such changes. The descriptors $\alpha, \mathrm{T}_{22}$, and $\mathrm{P}_{\mathrm{D}}$ appear to be better than any others for detecting bare land to building changes. If we consider the polarimetric components $\mathrm{HH}$ and $\mathrm{VV}$,

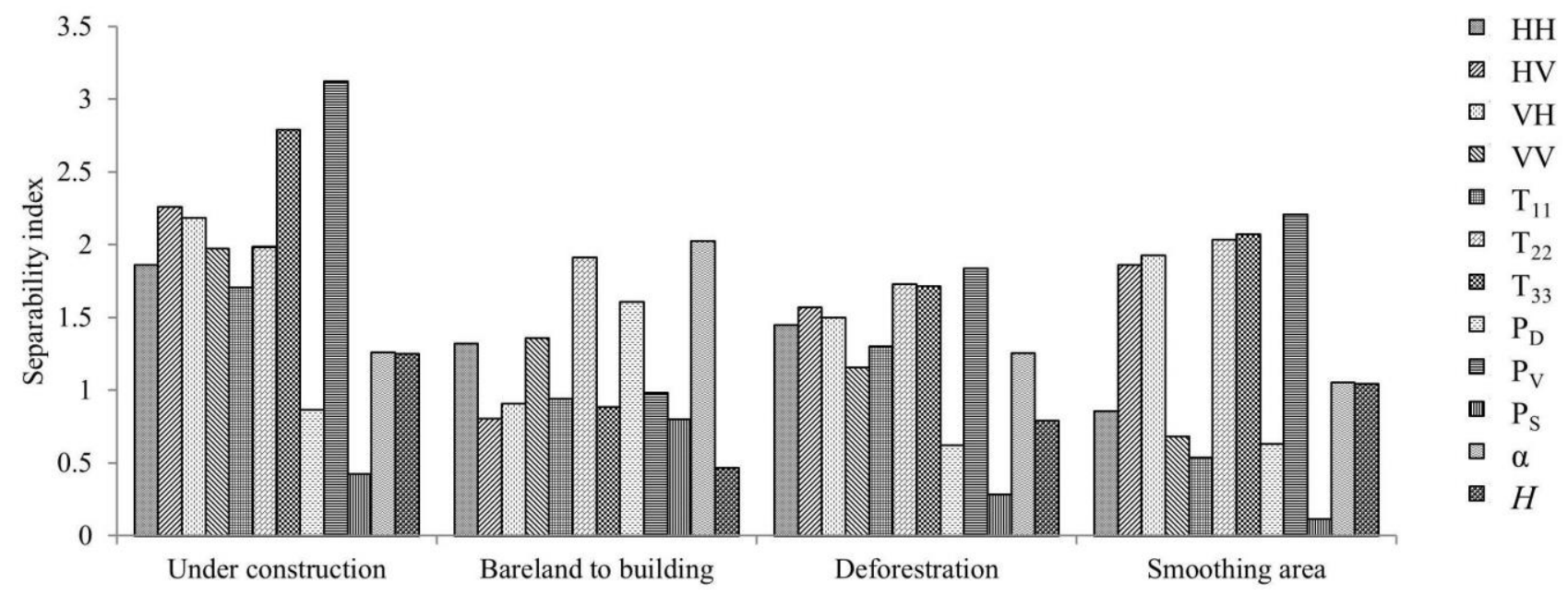

Fig. 9. Separability index of different polarimetric descriptors in major change classes. 
neither shows the highest separability index in any change class. However, they are moderately effective for each change.

Thus, from this analysis, what we can say is that a single descriptor cannot be equally effective for each type of change and some descriptors appear to be better for detecting specific types of change. Therefore, it is likely to improve the overall accuracy of change detection if we make use of complementary pairs: that is, use those polarimetric descriptors that have better separability indexes in a tight coupling. $\mathrm{P}_{\mathrm{V}}, \mathrm{T}_{33}$, and $\mathrm{HV}$ are more sensitive to three of the major types of change (agricultural to bare land, deforestation, and construction). Similarly, $\mathrm{T}_{22}, \mathrm{P}_{\mathrm{D}}$, and $\alpha$ are the most sensitive to changes in the dihedral structure. Hence, a complementary pair can be formed by taking one descriptor from each group.

2) Change Detection from Polarimetric Descriptors and Discussion

Fig. 10 shows change detection maps developed from pairs of polarimetric descriptors for April 2007 to April 2011 data. The accuracy assessment is summarized in Table 1. Change detection results obtained from $H$ and $\alpha$ are shown in Figs. 10 (c) and (d), respectively. The accuracy obtained from $H$ is slightly better than that from $\alpha$; however, the major sources of error are the same in both. Some specific change

Table 1. Comparison of urban and suburban change mapping accuracies from different polarimetric descriptors.

\begin{tabular}{|c|c|c|c|c|c|c|c|c|c|c|c|c|}
\hline Indicators & $\mathrm{HH}$ & $\mathrm{HV}$ & $\mathrm{VH}$ & $\mathrm{VV}$ & $\mathrm{T}_{11}$ & $\mathrm{~T}_{22}$ & $\mathrm{~T}_{33}$ & $P_{D}$ & $\mathrm{P}_{\mathrm{V}}$ & $\mathrm{P}_{\mathrm{S}}$ & $\alpha$ & $H$ \\
\hline $\begin{array}{l}\text { False alarm } \\
\text { rate }(\%)\end{array}$ & 11.96 & 10.27 & 10.52 & 14.55 & 13.83 & 9.60 & 10.28 & 12.92 & 9.41 & 23.81 & 19.89 & 17.20 \\
\hline $\begin{array}{l}\text { Increased } \\
\text { intensity }(\%)\end{array}$ & 84.60 & 78.67 & 78.97 & 83.53 & 79.56 & 89.19 & 72.72 & 59.29 & 75.72 & 63.85 & 82.43 & 86.99 \\
\hline $\begin{array}{l}\text { Decreased } \\
\text { intensity }(\%)\end{array}$ & 66.41 & 63.03 & 62.9 & 59.10 & 67.56 & 60.06 & 61.98 & 15.21 & 66.51 & 15.40 & 21.46 & 38.98 \\
\hline $\begin{array}{l}\text { Kappa } \\
\text { coefficient }\end{array}$ & 0.73 & 0.74 & 0.74 & 0.70 & 0.69 & 0.73 & 0.75 & 0.56 & 0.76 & 0.48 & 0.62 & 0.68 \\
\hline
\end{tabular}

False alarm rate $(\%)$ - Percentage of falsely classified pixels.

Increased intensity $(\%)$ - Percentage of correctly detected increased backscattering intensity areas.

Decreased intensity $(\%)$ - Percentage of correctly detected decreased backscattering intensitv areas.

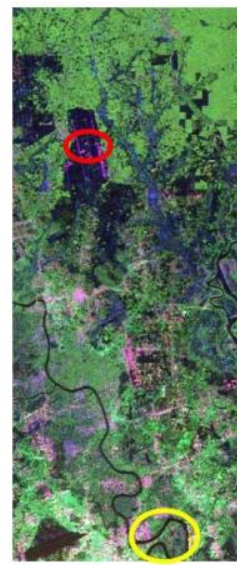

(a) Pauli 2007

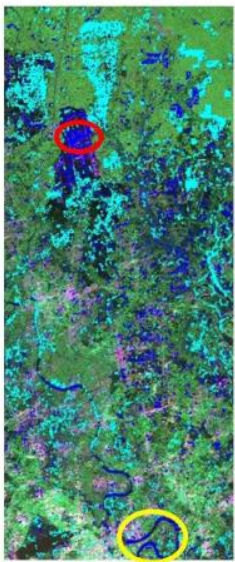

(h) VV

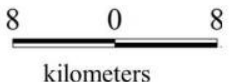

kilometers

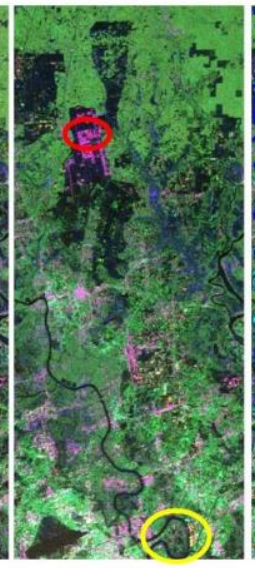

(b) Pauli 2011

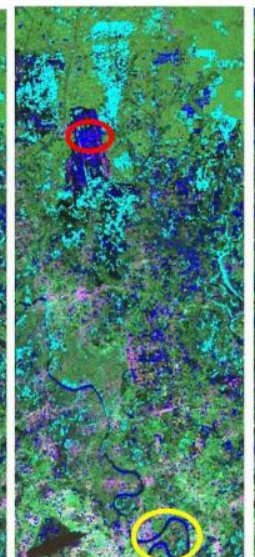

(i) $\mathrm{T}_{11}$

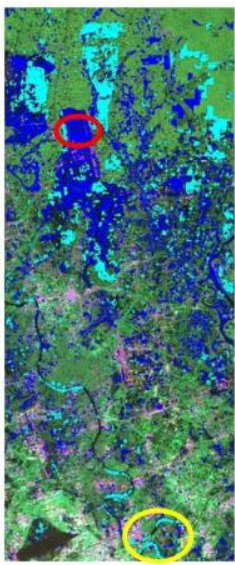

(c) $\alpha$

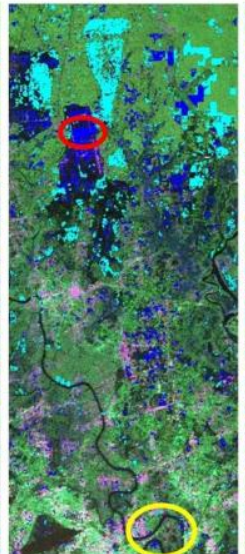

(j) $\mathrm{T}_{22}$

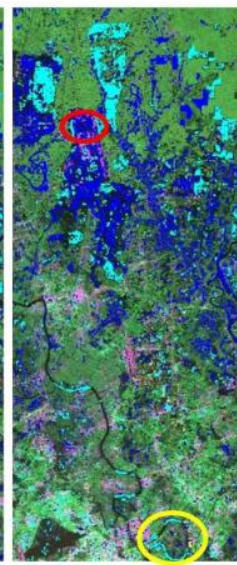

(d) $\mathrm{H}$

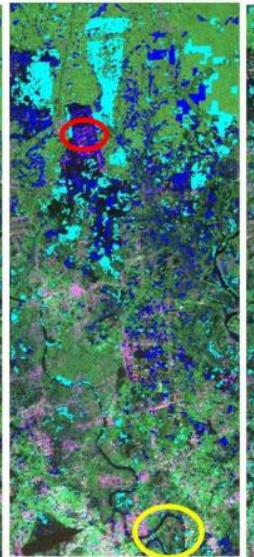

(k) $\mathrm{T}_{33}$

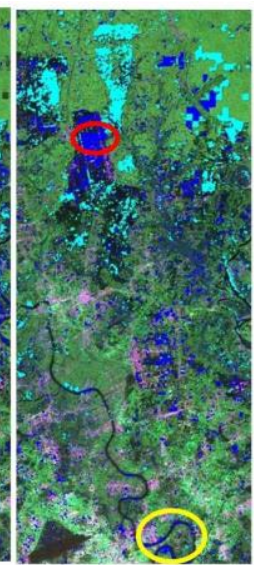

(e) $\mathrm{HH}$

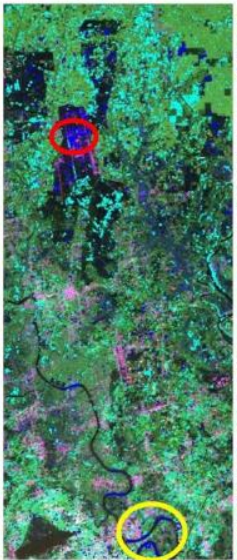

(1) $\mathrm{P}_{\mathrm{S}}$

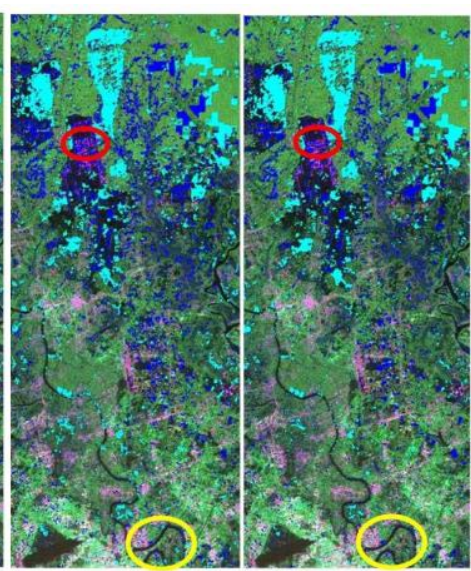

(f) $\mathrm{HV}$

(g) $\mathrm{VH}$

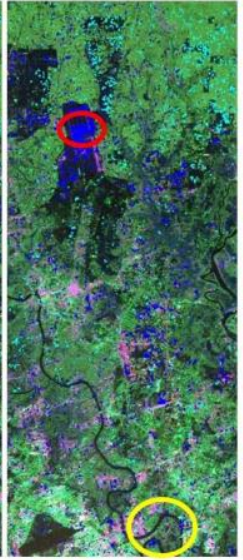

(m) $\mathrm{P}_{\mathrm{D}}$

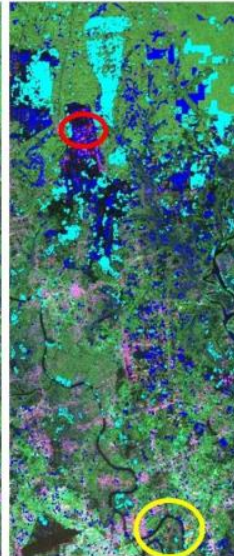

(n) $\mathrm{P}_{\mathrm{V}}$

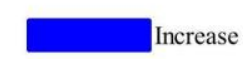

Decrease

Built-up

Water body-no change

Fig. 10. Change map obtained from polarimetric descriptors. (a) - (b) False color composite of Pauli vectors generated from PolSAR images in 2007 and 2011, and (c) - (n) change detection maps derived from the various polarimetric descriptors by supervised thresholding method, overlaid with Pauli vector false color composite. 
types, such as change from bare land to vegetation, are not detected by either descriptor, which leads to many missing alarms.

Figs. 10(e)-(h) indicate the changes in single channel backscattering measurements for $\mathrm{HH}, \mathrm{HV}, \mathrm{VH}$, and $\mathrm{VV}$ polarizations, respectively. The results obtained from the co-polarization descriptors ( $\mathrm{HH}$ and $\mathrm{VV}$ ) are very similar. Likewise, the results from $\mathrm{VH}$ component are very similar to those of the HV component. The cross-polarization descriptors (HV and $\mathrm{VH}$ ) give slightly different results from those obtained from the co-polarization channels. Some water bodies appear as change areas in $\mathrm{HH}$ and $\mathrm{VV}$, as shown by a yellow circle in Fig. 10. However, they are not changed in the reference map. Wind direction, turbidity, and movement in the water body can alter the backscattering in SAR images. Although these assumptions were not verified, we treated these areas as no-change areas.

Among the model-based decomposition components (Figs. 10 (1)-(n)), the change detection map obtained from $P_{V}$ exhibits the best detectability. The accuracy obtained from $P_{D}$ is not comparable with that from $\mathrm{P}_{\mathrm{V}}$ but it differs from $\mathrm{P}_{\mathrm{V}}$ in the change types detected and thus they appear to complement each other. For $\mathrm{P}_{\mathrm{S}}$, false and missing alarms are more frequent, but it can detect some changes that are not detected by $\mathrm{P}_{\mathrm{V}}$ or $\mathrm{P}_{\mathrm{D}}$. Some water bodies seen as changes are not really changed. The source of this false alarm is the same as for $\mathrm{HH}$ and $\mathrm{VV} . \mathrm{T}_{11}, \mathrm{~T}_{22}$, and $\mathrm{T}_{33}$ (Figs. 10 (i)-(k)) are nearly the model-based decomposition. $\mathrm{T}_{33}$ is very similar to $P_{V}$, but $T_{22}$ mixes results from $P_{S}$ and $P_{D}$. Even though it is close to $P_{D}, T_{22}$ possesses the detection capability in $P_{S}$ as well. This is because $T_{22}$ is used for computing $P_{S}$, along with other polarimetric descriptors from the coherency matrix. Additionally, $\mathrm{T}_{11}$ also has the capability to detect changes that cause changes in $P_{S}$ and $P_{D}$, but it is not as robust. This is because $\mathrm{T}_{11}$ is one of the major contributors in the computation of $P_{S}$ and $P_{D}$. The major source of error in $T_{11}$ is the same as in $P_{S}$.

The accuracy assessment confirms that the frequency of missing alarms in $P_{D}$ is significant. Decreased intensity areas, such as those with a change from agricultural land to bare land (smoothing area) and deforestation, are not sensitive to $\mathrm{P}_{\mathrm{D}}$, and thus have higher missing alarm rates. Similarly, several increased backscattering areas are under construction and thus clear dihedral structures have not been formed, resulting in significant changes in $\mathrm{P}_{\mathrm{V}}$ rather than $\mathrm{P}_{\mathrm{D}}$. However, the false alarm rate is very low in $P_{D}$, and $P_{D}$ is complementary to $\mathrm{P}_{\mathrm{V}}$ and $\mathrm{T}_{33}$. $\mathrm{T}_{22}$ is equally sensitive to the change from bare land to dihedral structures as $\mathrm{P}_{\mathrm{D}}$, but it is better than $P_{D}$ for other kinds of changes, such as agricultural land to bare land or deforestation.

$\mathrm{T}_{11}$ and $\mathrm{P}_{\mathrm{S}}$ have some common information and show different scattering characteristics than other descriptors in some changes for example, in the deforestation area, while $P_{v}$ is decreased, $P_{S}$ is increased. Additionally, a significant false alarm rate occurred in these descriptors may cause a decrease in the overall performance if they are used in fusion with other descriptors. Therefore, the fusion of $\mathrm{T}_{22}$ and $\mathrm{T}_{33}, \mathrm{HH}$ and $\mathrm{HV}$, and $\mathrm{P}_{\mathrm{D}}$ and $\mathrm{P}_{\mathrm{V}}$ would be worthwhile to detect the diverse changes occurring in an urban and suburban environment and reduce the rate of false and missing alarms. Moreover, $\mathrm{HH}$ and VV are interchangeable and, of course,

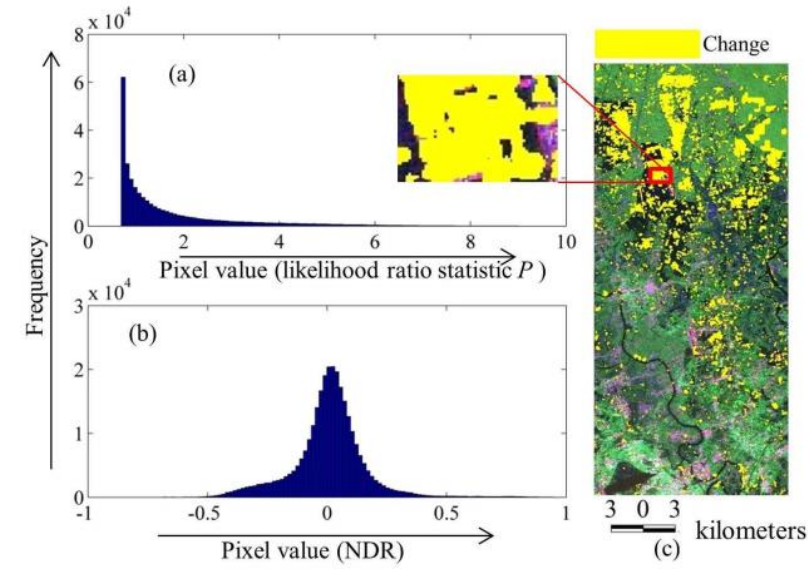

Fig. 11. Results from likelihood ratio statistic. (a) Histogram of the likelihood ratio statistic (P), (b) histogram of the NDR image generated from the $\mathrm{HH}$ component, and (c) the change map derived from the likelihood ratio statistic.

$\mathrm{HV}, \mathrm{T}_{33}$, and $\mathrm{P}_{\mathrm{V}}$ can replace each other. The accuracy of urban change detection by $H$ and $\alpha$ is fairly good. However, the increase and decrease in $H$ and $\alpha$ do not have exactly same meaning as increased and decreased backscattering from other polarimetric descriptors. Thus, they cannot be used to complement any other polarimetric descriptors.

In addition to single polarimetric descriptors, a polarimetric similarity test between two images was implemented. The likelihood ratio test statistic $P$ can be given as.

$$
P=\ln \left(\frac{\left|C_{\text {avg }}\right|}{\left|C_{1}\right|}\right)+\ln \left(\frac{\left|C_{\text {avg }}\right|}{\left|C_{2}\right|}\right)
$$

where $C_{1}$ and $C_{2}$ are the covariance matrices corresponding to the images acquired at time 1 and time 2, respectively, and $C_{a v g}$ is $\left(C_{1}+C_{2}\right) / 2$. This test statistic has the Wishart distribution [3], [18], [19].

Fig. 11(c) shows the change map developed from the likelihood ratio test statistic $P$. The overall kappa coefficient is 0.79 , the overall detectability is $83 \%$ with a false alarm rate of $12 \%$. The results are better than those of a single polarimetric descriptor, but they also fail to solve the false alarm problem for an unchanged water body. In addition to that, this technique is not very sensitive to changes from agricultural land to bare land, which causes a very small change in the overall scattering mechanism that is dominated by $\mathrm{HV}\left(\mathrm{P}_{\mathrm{V}}\right)$. The major disadvantages in this method arise from the single-tail curve generated by the likelihood ratio test statistic, as shown in Fig. 11(a). A single threshold value will distinguish change and no-change areas. Thus, it entails two types of deficiency. The first problem is that it can only distinguish between change and no-change areas, but not between increased and decreased backscattering. The second problem is the misclassification error. This arises because the change area is not symmetrical, as shown in Fig. 11(b). When converting such data into a single-tail statistic, the nature of the overall curve will differ from both tails, and a single threshold value cannot perfectly judge both types of change. That is, what is good for one type of change (increased or decreased backscattering) will not be good for the other. In this case, a higher missing alarm rate in detecting decreased backscattering areas was identified. 


\section{B. Change Mapping Using Complementary Pairs of Polarimetric Descriptors}

The polarimetric scattering mechanism analysis and change detection results obtained from different descriptors imply that several polarimetric descriptors provide complementary information about changes. Therefore, the performance of change detection can be improved by the fusion of several polarimetric descriptors. From the sensitivity analysis, three independent sets of complementary couple namely $\{\mathrm{HH}, \mathrm{HV}$ ( $\mathrm{VH}$ is considered equivalent to $\mathrm{HV})\},\left\{\mathrm{T}_{22}, \mathrm{~T}_{33}\right\}$, and $\left\{\mathrm{P}_{\mathrm{D}}, \mathrm{P}_{\mathrm{V}}\right\}$ were identified. Each member of each set is complementary to the other member of the same set and they are complete, i.e. can detect all changes that are sensitive to SAR backscatter. From the analysis, it is observed that $\mathrm{HH}$ and $\mathrm{VV}$ have similar responses to the various changes, therefore they may be used interchangeably. In addition, spatial information is also useful to improve change detection performance and so is considered here.

Thus, the results from using each of the three complementary pairs of polarimetric descriptors were evaluated independently. Fig. 12 shows the resulting change detection maps, and Table 2 gives the corresponding mapping accuracy.

As all the descriptors come from the same data sources, the detected results do not seem to differ much visually. The overall accuracy assessment, based on the Kappa coefficient, shows that the results obtained from the fusion of $\mathrm{T}_{22}$ and $\mathrm{T}_{33}$ are better than all other fusion results. The unchanged water body that is detected as a changed area by the fusion of $\mathrm{HH}$ and $\mathrm{HV}$ is the main source of the increase in the false alarm rate. Similarly, the changed features that cause changes in surface scattering are missing when using $\mathrm{P}_{\mathrm{D}}$ and $\mathrm{P}_{\mathrm{V}}$ only. In contrast to that, the fusion of $\mathrm{T}_{22}$ and $\mathrm{T}_{33}$ overcomes both the false alarm and missing alarm problems. The false detection of the water body was not seen in any of the $T_{22}$ and $T_{33}$ results. Additionally, as discussed in the previous section, it is $T_{22}$, that possesses the potential to detect the same changes as $\mathrm{P}_{\mathrm{S}}$ and is responsible for the improvement of accuracy in the fusion of $\mathrm{T}_{22}$ and $\mathrm{T}_{33}$.

The fusion of polarimetric descriptors improves the results in two ways, as we carried it out in two steps. The union operator is responsible for overcome the missing alarm problem by making use of the complementary characteristics of several descriptors. In addition, the classification of ambiguous pixels identified by modified thresholding and the union operation was done successfully through the

Table 2. Change mapping accuracies with the fusion of several complementary sets of polarimetric descriptors.

\begin{tabular}{|l|l|l|l|}
\hline Indicators & $\mathrm{HH} \& \mathrm{HV}$ & $\mathrm{T}_{22} \& \mathrm{~T}_{33}$ & $\mathrm{P}_{\mathrm{D}} \& \mathrm{P}_{\mathrm{V}}$ \\
\hline $\begin{array}{l}\text { False alarm rate } \\
(\%)\end{array}$ & 8.52 & 6.50 & 7.26 \\
\hline $\begin{array}{l}\text { Increased } \\
\text { intensity (\%) }\end{array}$ & 93.20 & 93.92 & 92.57 \\
\hline $\begin{array}{l}\text { Decreased } \\
\text { intensity (\%) }\end{array}$ & 79.19 & 80.14 & 78.05 \\
\hline $\begin{array}{l}\text { Kappa } \\
\text { coefficient }\end{array}$ & $\mathbf{0 . 8 1}$ & $\mathbf{0 . 8 5}$ & $\mathbf{0 . 8 3}$ \\
\hline
\end{tabular}

False alarm rate (\%) - Percentage of falsely classified pixels.

Increased intensity $(\%)$ - Percentage of correctly detected increased backscattering intensity areas.

Decreased intensity (\%) - Percentage of correctly detected decreased backscattering intensity areas.

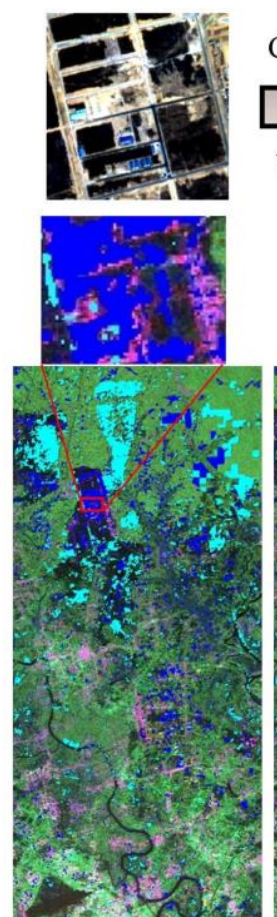

(a) $\mathrm{HH} \& \mathrm{HV}$

$\begin{array}{lll}3 & 0 & 3\end{array}$

- kilometers
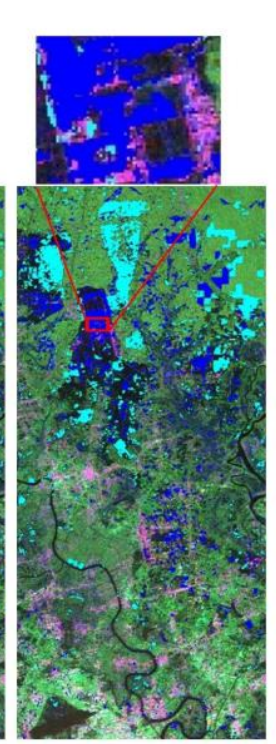

(b) $T_{22} \& T_{33}$

Decrease

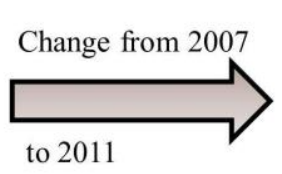

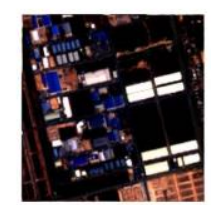
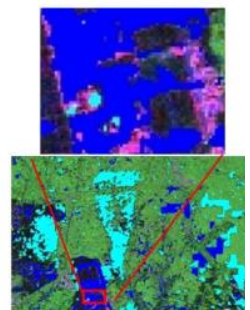

(c) Dbl. \& Vol.
Fig. 12. Change map obtained from the proposed fusion method. (a) $H H \& V V$, (b) $T_{22} \& T_{33}$, (c) $P_{D} \& T_{V}$, overlaid in a false color composite of Pauli vectors.

region-growing algorithm, which was modified to use local information from all polarimetric descriptors considered in the fusion process. Thus, the accuracy of mapping large areas of urban change was notably improved, with a significant reduction in the missing and false alarm rates, by the fusion of complementary descriptors.

\section{V.CONCLUSIONS AND RECOMMENDATIONS}

Changes in the radar scattering mechanism for urban and suburban areas were investigated by using several polarimetric descriptors. Changes in radar backscattering from built-up areas are characterized by significant changes in $P_{D}$ due to the presence of dihedral structures. $T_{22}$ is also highly sensitive to these changes. All other descriptors revealed the same changes; however, they are not as effective. Deforestation is characterized by a significant decrease in $\mathrm{P}_{\mathrm{V}}$. In contrast, $\mathrm{P}_{\mathrm{V}}$ was found to increase for built-up areas in which the structures were not clearly dihedral, such as buildings under construction, small houses with sloped roofs, and structures not aligned to the image orientation. In terms of separability, $\mathrm{P}_{\mathrm{V}}$ and its family (HV and $\mathrm{T}_{33}$ ) are sensitive to three major changes: deforestation, construction, and change from bushes or agricultural land to bare land. The descriptors $H$ and $\alpha$ could identify changed areas; however, they were not able to distinguish clearly between areas of increased and decreased intensity. Moreover, they do not have any complementary descriptors.

A supervised change detection approach was applied to various polarimetric descriptors. The accuracy assessment results indicated that single polarization observation can give a kappa coefficient up to 0.74 , and a single polarimetric descriptor up to 0.76 . These results match the characteristics obtained from separability index. Thus, the fusion of 
polarimetric descriptors is important to optimize performance.

The major aim of this research is to obtain a change map that allows the separation of both types of change areas (increased and decreased intensity) from unchanged background. To achieve this, a new polarimetric information fusion approach based on the coupling of thresholding and a region-growing algorithm was implemented for several complementary pairs of descriptors. The contextual fusion of $\mathrm{T}_{22}$ and $\mathrm{T}_{33}$ gave the best change detection results for urban and suburban environments, with a kappa coefficient of 0.85 and a significant improvement in the false and missing alarm rates.

The supervised thresholding algorithm adopted in this study was tested for only ordinary changes and not for changes resulting from a disaster in which most of the area could be affected by the disaster and it may be difficult to find an appropriate no-change sample. Therefore, we strongly recommend caution when considering the use of this supervised thresholding algorithm to monitor disaster effects. Additionally, the different scattering characteristics appear in surface scattering seem to be very useful for the automatic change pattern detection and is a promising research topic. Moreover, it should be noted that apparent changes might occur in water bodies due to turbidity or different air directions. Users should carefully examine any changes to a water body.

\section{ACKNOWLEDGMENT}

This research was supported in part by a program of the 4th ALOS-2 research announcement of the Japan Aerospace Exploration Agency (JAXA). The authors are thankful to anonymous reviewers for their constructive criticism.

\section{REFERENCES}

[1] Y. Bazi, L. Bruzzone, and F. Member, “An unsupervised approach based on the generalized Gaussian model to automatic change detection in multitemporal SAR images", IEEE Trans. Geosci. Remote Sens., vol. 43, no. 4, pp. 874-887, 2005.

[2] G. Moser and S. B. Serpico, "Generalized minimum-error thresholding for unsupervised change detection from SAR amplitude imagery", IEEE Trans. Geosci. Remote Sensi., vol. 44, no. 10, pp. 2972-2982, 2006

[3] S. Park., Yamaguchi Y. and Kim D., "Polarimetric SAR remote sensing of the 2011 Tohoku earthquake using ALSO/PALSAR", Remote Sens. Envt., vol. 132, pp. 212-220, 2013.

[4] E.J.M. Rignot, and J.J. Van Zyl, "Change detection techniques for ERS 1 SAR data”, IEEE Trans. Geosci. Remote Sens., vl. 31, pp. 896-906, 1993.

[5] W. Dierking and H. Skriver, "Change detection for thematic mapping by means of airplane multitemporal polarimetric SAR imagery", IEEE Trans. Geosci. Remote Sens., vol. 40, no.3, pp. 618-636, 2002.

[6] Y. Ban and O.A. Yousif, "Multitemporal space borne SAR data for urban change detection in China", IEEE journal of selected topics in applied earth observations and Remote Sens., vol. 5, no. 4, pp. 1087-1094, 2012.

[7] Y. Yamaguchi, A. Sato, W. Boerner, R. Sato, and H. Yamada, "Four component scattering power decomposition with rotation of coherency matrix", IEEE Trans. Geosci. Remote Sens., vl. 49,no. 6, pp. 2251-2258, 2011.

[8] S.R. Cloude, and E. Pottier, "An Entropy Based Classification Scheme for Land Applications of Polarimetric SAR", IEEE Trans. on Geosci. Remote Sens., vol. 35, no. 1, 68-78, 1997.

[9] A. Freeman, and S. L. Durden, "A three component scattering model for polarimetric SAR data”, IEEE Trans. Geosci. Remote Sens., vol. 36, no. 3, pp. $963-973,1998$.
[10] ASF MapReady User Manual, Version 3.1, Alaska Satellite Facelity Engineering Group, 2013, pp. 14-62.

[11] A. Lopes, R. Touzi, and E. Nezry, "Adaptive Speckle Filters and Scene Heterogeneity," IEEE Transactions on Geoscience and Remote Sensing, vol. 28, no. 6, pp. 992-1000, Nov. 1990.

[12] B. Mishra, and J. Susaki, "Coupling of thresholding and a region growing algorithm for change detection in SAR images", Progress in Electromagnetic Researches, vol. 143, pp. 519-544,3013.

[13] P.R. Coppin and M.E. Bauer, "Processing of multitemporal Landsat TM imagery to optimize extraction of forest cover change feature", IEEE Trans. Geosci. Remote Sens., vol. 30, no. 4, pp. 918-927, 1994.

[14] M. Gianinetto and P. Villa, "Mapping Hurricane Katrina's widespread destruction in New Orleans using multsensor data and the normalized difference change detection (NDCD) technique", Internal J. Remote Sens., vol. 32, no. 7, pp. 1961-1982, 2011.

[15] E. J. M. Rignot and J. J. van Zyl, "Change detection techniques for ERS-1 data", IEEE Trans. Geosci. Remote Sens., vol. 31, no. 4, pp. 896-906, 1993.

[16] P. Mishra, D. Singh, and Y Yamaguchi "Land cover classification of PALSAR images by knowledge based decision tree classifier and supervised classifiers based on SAR observables", Progress in Electromagnetics Research B, vol. 30, pp. 47-70, 2011.

[17] A. Singh, "Review Article Digital change detection techniques using remotely-sensed data," Int. J. Remote Sens., vol. 10, no. 6, pp. 989-1003, Jun. 1989.

[18] K. Conradsen, A. A. Nielsen, J. Schou, and H. Skriver, "A test statistic in the complex Wishart distribution and its application to change detection in polarimetric SAR data", IEEE Trans. Geosci. Remote Sens., vol. 41, no.1, pp. 4-19, 2003.

[19] P. Kersten, J. S. Lee, and T. Ainsworth. "A comparison of change detection statistics in POLSAR images", Proceedings of IEEE Geoscience and Remote Sensing Symposium 2005, July 25-29, Seoul, Korea, pp. 4836-4839, 2005.

[20] N. Otsu, "A Threshold Selection Method from Gray-Leve Histogram”, IEEE Trans. Systems Man, and Cybernetics, Vol. 9, pp 62-66, 1979.

[21] L.C. Altorre, R. Sanchez-Andres, S. Cirujano, S. Begueria, and S. Sanchez-Carrillo. "Identification of Mangrove Areas by Remote Sensing: The ROC Curve Technique Applied to the Northwestern Mexico Coastal Zone Using Landsat Imagery", Remote Sens, Vol. 3, pp. 1568-1583, 2011. 